\title{
The role of magnetic sensors in the hybridisation and electrification of vehicles
}

\author{
Marc Kramb ${ }^{1 *}$, and Rolf Slatter ${ }^{1}$, \\ 'Sensitec GmbH, Georg-Ohm-Str. 11, 35633 Lahnau, Germany
}

\begin{abstract}
Electrical currents need to be measured in a wide variety of different applications in the field of power electronics. However, the requirements for these measurement devices are becoming steadily more demanding regarding accuracy, size and especially bandwidth. In order to increase the power density of power electronics, as particularly important in the field of electromobility, there is a clear causal chain. Soft switching leads to higher efficiency and higher frequencies, which enable smaller dimensions for a given power output. Higher switching frequencies allow the size of magnetic components to be reduced significantly, resulting in more compact and lighter designs. This trend is now being reinforced by use of new wide bandgap semiconductor materials like silicon carbide ( $\mathrm{SiC})$ and gallium nitride $(\mathrm{GaN})$, as their low on-resistances and low parasitic capacitances reduce switching losses. Conventional current sensor solutions, e.g. hall- or shunt based sensors exhibit a limited bandwidth, typically less than $250 \mathrm{kHz}$. Other current sensors, like those based on the Rogowski-Coil, are capable of highly dynamic current measurement, but are significantly more expensive, larger and hence not suitable for large series applications. Furthermore, Rogowski-Coils are only capable of measuring alternating currents (AC), which prevents their use in applications where DC currents must also be measured. In order to meet the above mentioned requirements, magnetoresistive (MR) current sensors are ideally suited due to the fact that the bandwidth of the magnetoresistive effect extends up into the GHz-range. This paper describes the principle of operation and main performance characteristics of highly integrated MR current sensors and describes their benefits compared to other types of current sensor, in particular with regard to applications in the hybridisation and electrification of vehicles.
\end{abstract}

\section{Introduction}

The magnetoresistive effect has a long history, the Anisotropic MagnetoResistive (AMR) effect being first discovered in 1857 by Lord Kelvin. The AMR effect occurs in ferromagnetic materials, such as nickel-iron layers structured as strip elements, whose specific impedance changes with the direction of an applied magnetic field. Due to a special structure of the strips the resistance change is proportional to the applied magnetic field over a wide range. This means that by adept design of the sensor structure very small magnetic fields can be detected with very high accuracy. However, the MR-effect did not experience widespread use until the early 1980s, when the first MR-based read heads were implemented in hard disc drives. The first industrial applications for MR-based sensors followed at the beginning of the 1990s, since when the number of applications has increased dramatically. MR sensors are used extensively in safety-critical automotive applications, for example in wheel speed sensors for the ABS-system or in steering angle sensors for the ESP-system.

The magnetoresistive effect is particularly attractive in the field of electrical current measurement. The very high sensitivity means that there is no need to use an iron core to concentrate the magnetic field generated by the conductor carrying the current. This means that MR-based current sensors do not suffer from hysteresis and that they have a significantly higher bandwidth, enabling current sensors with bandwidths in the $\mathrm{MHz}$ area possible. Compared to shunt resistors MR-based sensors have the benefit of galvanic isolation and dramatically lower power losses. This is particularly important in high voltage applications and where overall power efficiency is a major design driver like in the electrification of vehicles. 


\section{Anisotropic MagnetoResistive Effect (AMR)}

In materials that exhibit anisotropic magnetoresistance the resistance depends on the angle between the current direction and magnetization direction. The resistance is maximal when the current and magnetization directions are parallel, minimum when perpendicular (Figure 1).

The characteristic of the simple thin film MR strip is non-linear, the direction of the magnetic field is not determinable and the sensitivity approaches zero for small field strengths. Conducting stripes typically made of aluminium are deposited at $45^{\circ}$ to the anisotropy axis (Barber pole structures) serve to linearize the characteristic of the MR strip (Figure 2).

Fig. 1: AMR Effect

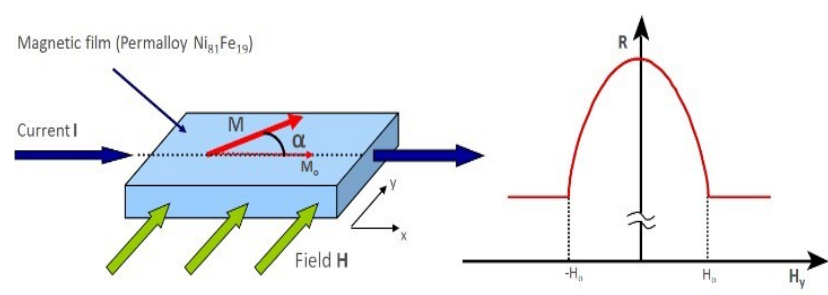

Fig. 2: Influence of Barber poles

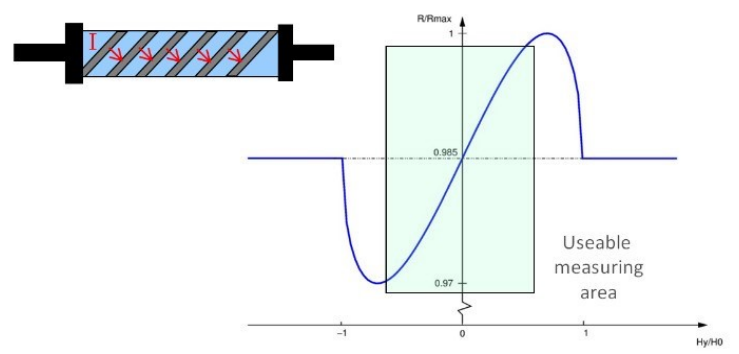

\section{$3 \quad$ Highly integrated current sensing}

Sensitec has a long experience of developing MR-based current sensors for industrial applications. It has been shown that the AMR current sensor principle is able to measure currents at very high frequencies [1]. The step response time is in comparison to a state of the art sensors significantly shorter (Figure 3).

The increased demand for very compact current sensors generated by the recent trend to electromobility was the driver for the development of a highly integrated current sensor (CFS1000) comprising an AMR sensor chip, a signal conditioning ASIC (application-specific integrated circuit) and two biasing permanent magnets (Figure 4). The latter are necessary for maintaining the initial magnetization direction of the AMR structures in the case of overcurrent situations. A special lead-frame as well as an advanced assembly technique enables a "system-in-package" solution (SIP): all system components are molded within a JEDEC compliant SOIC16 package (Figure 4). The ASIC solution is a compromise between possible fast current sensing (Figure 3 ) and small yet cost effective devices.

Fig. 3: Step response comparison CMS3000 vs. standard current sensor

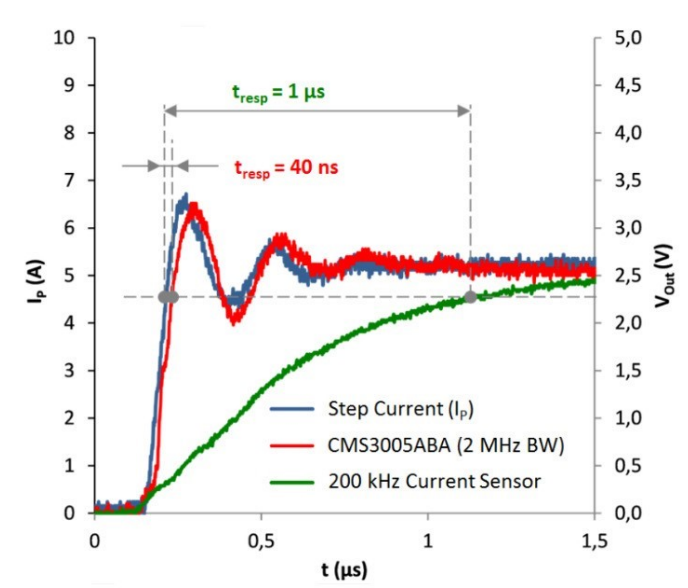

Fig. 4: CFS1000 current sensor concept

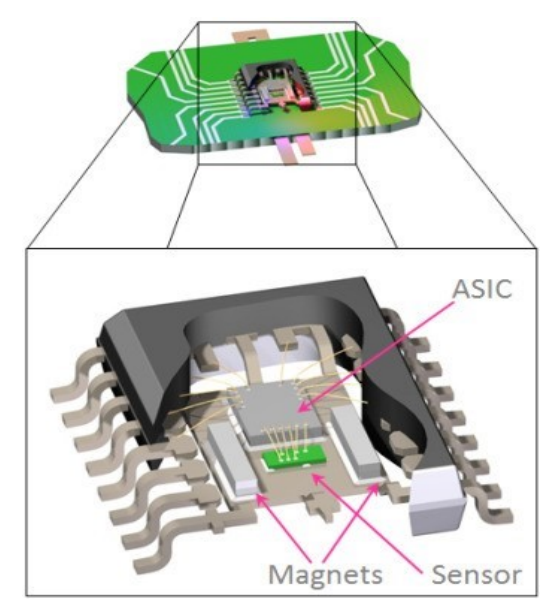


The product can physically be mounted with standard pick-and-place equipment onto a PCB and subsequently reflow soldered. The quantity to be measured is a differential magnetic field, also referred to as field gradient that is generated by two currents with opposed current flow directions. The primary current conductor is typically U-shaped, with its straight parallel parts positioned underneath the sensor (Figure 4, upper part).

For current measurement four AMR "resistors" are connected to form a Wheatstone bridge. The resistors on the silicon chip are placed so that they constitute a differential field sensor. This is necessary because interference fields can be eliminated this way. Combined with a signal conditioning and processing ASIC the chip is assembled on a substrate, which can be a hybrid circuit or a lead frame (Figure 5).

Furthermore, a compensation conductor is integrated on the chip with which a magnetic field can be generated close to the resistors. On the opposite side of the substrate the primary current conductor is attached below the MR chip in a $\mathrm{U}$-shape. The geometry of the primary conductor defines the measurement range of the current sensor. Based on the output signal from the MR chip, the ASIC (shown in Figure 5 schematically as an operational amplifier) generates a current Icomp in the compensation conductor, which compensates the magnetic field generated by the primary conductor in the plane of the AMR resistors. With this method the signal achieves a high linearity $(0.1 \%)$ and is largely independent of temperature. This compensation current is directly proportional to the primary current to be measured and is used to generate the output signal from the current sensor.

Fig. 5: Principle of operation

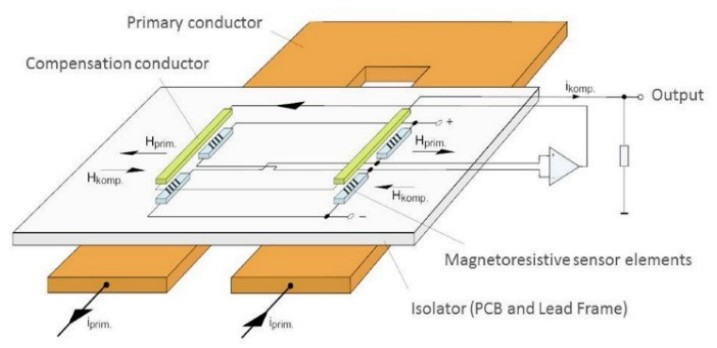

This "closed-loop" principle results in an extremely compact sensor that is largely insensitive to homogeneous interference fields and temperature changes, with a low power consumption and very high efficiency.

The AMR sensor chip (Figure 6) is designed as a differential field sensor in order to eliminate the effects of homogeneous disturbing fields such as are often encountered in power electronics applications.

Fig. 6: AMR current sensor chip layout

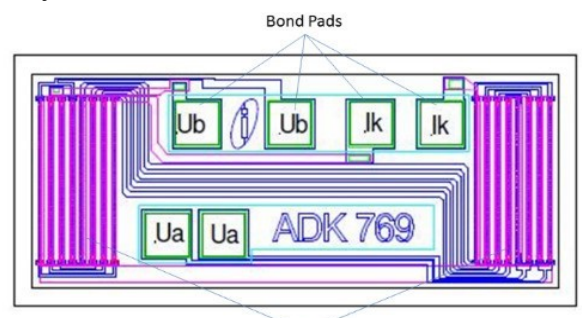

AMR Stripes with Barber Pole Structures

Figure 7 shows the location of the chip relative to the U-shaped primary conductor bar.

Fig. 7: Elimination of homogeneous stray fields

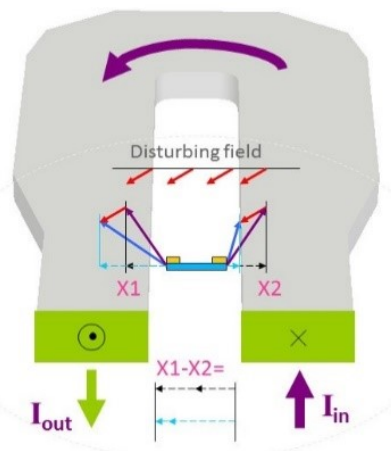


The sensitive areas of the chip only measure the horizontal component (X1 and X2) of the magnetic field generated by the current flowing in the U-shaped conductor. A disturbing field will decrease this component for one side of the chip, but increase it by an identical amount on the other side of the chip. The differential measurement principle means that the difference of the two components remains the same, so eliminating the influence of the disturbing field. In most applications there is therefore no need for additional shielding as is often necessary for surface mounted Halleffect current sensors with external current bar. The AMR-based current sensor exhibits no hysteresis as observed in iron core based Hall-sensor solutions and no remaining magnetic offset after overcurrent events. Due to the high sensitivity of the AMR sensor chip, a flux concentrator is not necessary. By variation of the geometry of the external primary current bar, the CFS1000 can be adapted to different current ranges and applications. With this, only one sensor type is needed for a wide range of applications. As an application example, an evaluation board with three current sensors for a nominal current of 200A was attached to an IGBT power module as can be seen in Figure 8.

Fig. 8: 3-phase module with CFS1000

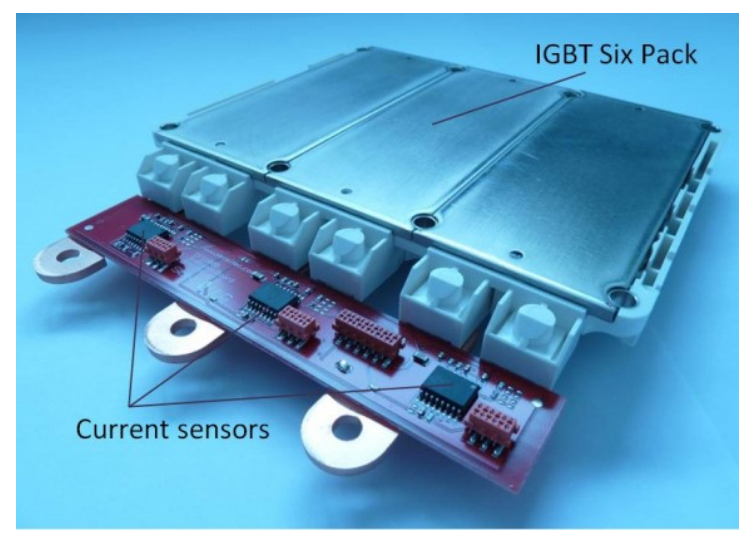

The system accuracy can be improved by adjusting both offset and sensitivity in the target application of the customer and by using either the internal or an external reference voltage. The adjustable over-current detection enables fast response in overload situations to prevent damage to the power transistors. A more detailed description of the principle of operation and the advantages of the CFS1000 can be found in [1]. Extensive tests have been undertaken as part of the AECQ100 qualification for automotive applications.

\section{$4 \quad$ Application Examples}

In a regularly updated roadmap for power density the Fraunhofer Institute for Integrated Systems and Device Technology (IISB) documents the trend to steadily increasing power density for drive inverters and DC/DC converters (Figure 9) [2]. The influence of wide band-gap devices is clearly visible from this trend. One particularly interesting application field for high-speed current sensing arises for DC/DC converters. The requirements for the bandwidth is rising into the $\mathrm{MHz}$ range in order to increase the power density. But also in safety critical applications, e.g. in traction inverters, where overcurrent situations need to be detected in the ns-range, extremely fast current sensors are necessary. To fulfill all these requirements, compact current sensors are necessary that detect currents highly dynamically, accurately and that are cost-effective.

Fig. 9: Benchmark designs and power current sensors density roadmap (Source: Fraunhofer IISB)

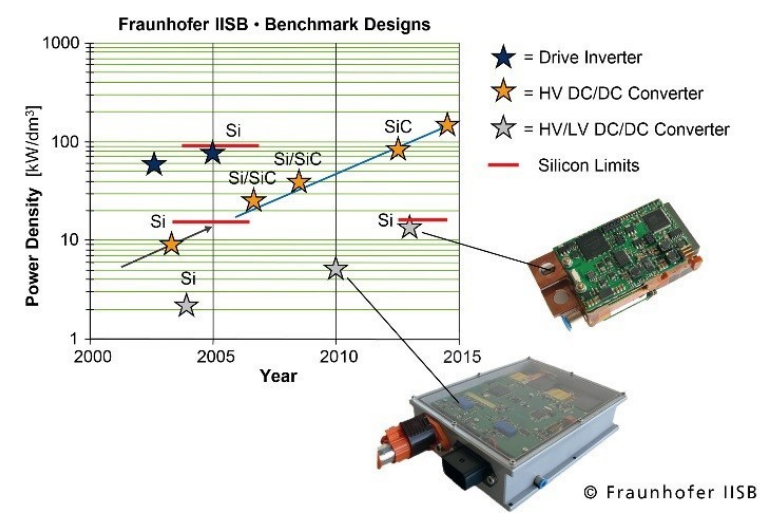




\subsection{DC/DC converter for e-mobility}

Within the BMBF research project PELiKAn the task was to develop a compact $2.5 \mathrm{~kW}$ DC/DC converter in order to replace the electric generator in automotive applications [3]. The DC/DC converter reduces the voltage of the high voltage batteries in hybrid and electric vehicles from $250 \mathrm{~V}-400 \mathrm{~V}$ down to the traditional $12 \mathrm{~V}$ supply voltage. High power efficiency could be realized as well as a light and compact design resulting in a power density of $14.3 \mathrm{~kW} / 1$ and dimensions of just $85 \mathrm{~mm} \times 50 \mathrm{~mm} \times 41 \mathrm{~mm}$. A key enabler is the small integrated magnetoresistive current sensor (CFS1000) that measures the output current of the DC/DC converter of up to $180 \mathrm{~A}$. In order to generate the differential magnetic field a compact U-shaped bus bar is designed and implemented into the output current line (Figure 10).

Fig. 10: DC/DC converter with U-shaped

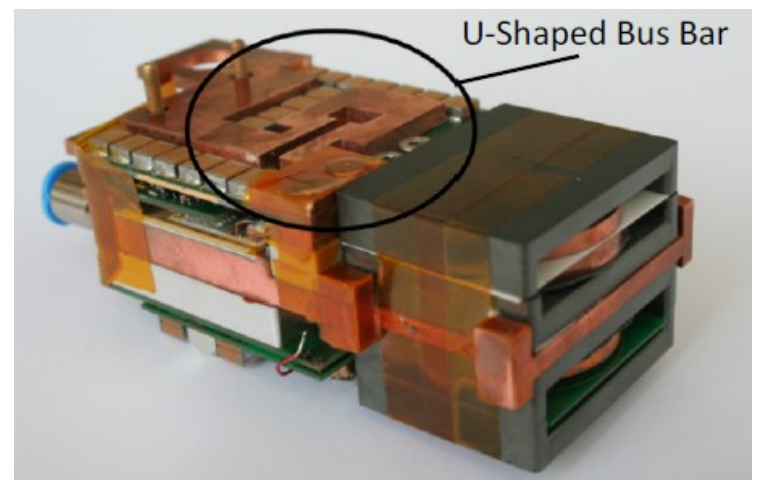

Fig. 11: CFS1000 attached to controller bus bar for 180 A rated current board (Source: Fraunhofer IISB) (Source: Fraunhofer IISB)

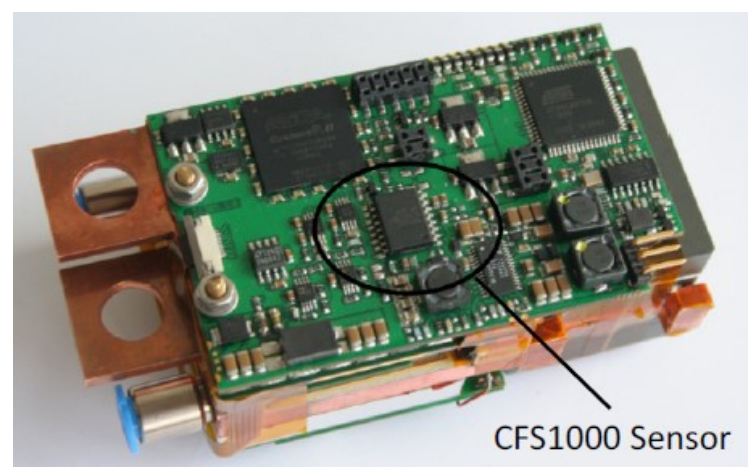

The current bar is attached to the backside of the control board. In Figure 11 the SOIC16 package is shown that is mounted on the front side of the PCB adjacent to other electrical components. The particular advantages of the CFS1000 current sensor for this application are its small SMT package to reduce volume and weight of the complete power electronics and the insensitivity to external stray fields that eliminates the need for additional external shielding.

\subsection{Drive inverter for hybrid commercial vehicle}

Current sensors based on the MR-effect have also played an important role in the EU-funded project COSIVU, where a number of suppliers and universities have worked together with the vehicle manufacturer Volvo to develop extremely compact power electronics and motors for a hybrid truck [4], [5]. The main objectives and highlights of the COSIVU project, which ran from 2012 to 2015 , can be summarized as follows:

- Decentralized drive-train system, managed by a central vehicle computer, for reduced weight and cooling complexity and thus improved energy efficiency

- One compact system package due to increased level of mechatronic integration for the electric motor

- Development of next generation of highly integrated inverter modules based on novel SiC technology $(1200 \mathrm{~V}, 500 \mathrm{~A})$ with innovative cooling concepts (e.g. double sided cooling), being capable of reducing energy losses by $>50 \%$

- Benchmarking of wireless communication between drive units and central computer vs. wired based solutions with respect to EMC issues, costs and durability 
- Implementation of innovative functional and health monitoring ( $\mathrm{SoF} / \mathrm{SoH})$ features, like thermal impedance spectroscopy for SoF/SoH determination of the inverter module or structure-borne sound analysis for $\mathrm{SoF} / \mathrm{SoH}$ determination of the motor/gear module

Figure 12 shows the basic concept for the decentralized drive-train, where the modular inverter for each actuator is mounted directly within the motor housing (Figure 13). This concept, called "site-of-action integration" greatly reduces the complexity and weight of the high voltage wiring harness. It also reduces the effort necessary to cool the various power modules and furthermore reduces the effort for EMI filtering [6].

Fig. 12: New electric drive topology

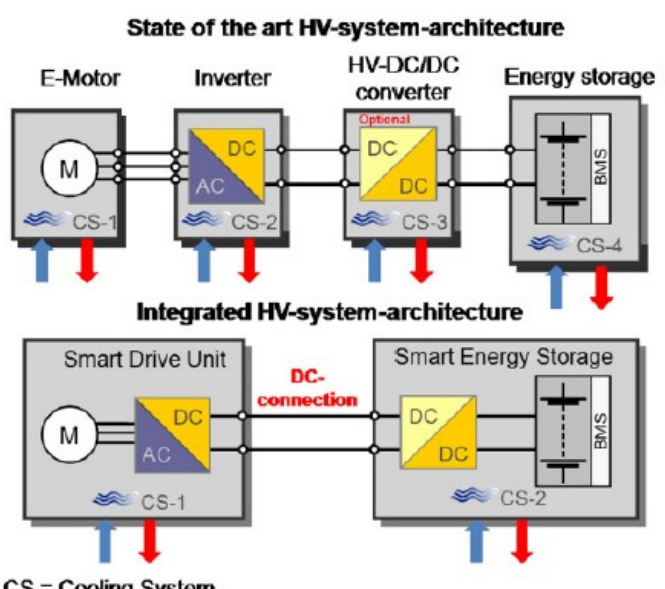

Fig. 13: COSIVU Drive system (Source: (Source: Fraunhofer IISB) Swerea IVF)

Fast, accurate current sensors are an important enabling technology for the high power density inverters essential for the implementation of this concept. Early in the project the partners reviewed the available technologies for current sensors, as summarized in Table 1 below. All other sensor technologies had significant disadvantages that prevented their use in this application and MR-based current sensors were selected as offering the best overall solution.

Table 1: Comparison of current sensor technologies

\begin{tabular}{|c|c|c|c|c|c|c|}
\hline Technology & Size & $\begin{array}{c}\text { Dynamic } \\
\text { performance }\end{array}$ & Accuracy & $\begin{array}{l}\text { Power } \\
\text { losses }\end{array}$ & Costs & \begin{tabular}{|l} 
Effort for \\
Design-In
\end{tabular} \\
\hline $\begin{array}{l}\text { Hall, Fluxgate } \\
\text { (closed loop) }\end{array}$ & -- & - & + & - & -- & + \\
\hline $\begin{array}{c}\text { Hall } \\
\text { (open loop) }\end{array}$ & ++ & - & -- & + & ++ & - \\
\hline Shunt & + & -- & ++ & -- & - & - \\
\hline Magnetoresistive & + & ++ & + & + & + & - \\
\hline
\end{tabular}

A particularly interesting result of the COSIVU project was the development of "building blocks" for the modular inverter. This low-impedance commutating cell provides the required robustness, EMC-compatibility and high efficiency through low switching losses (Figure 14).

The inverter building block (IBB) incorporates the DC-link capacitor, base driver, current sensor and direct cooled baseplate [6].

Fig. 14: Inverter Building Block (IBB) for Hybrid Commercial Vehicle (Source: Fraunhofer IISB)

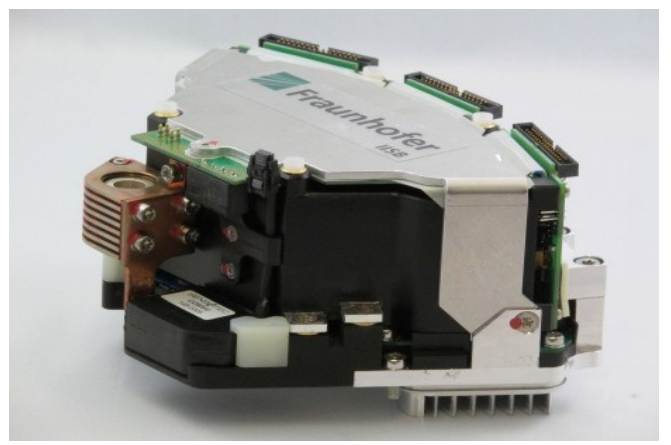


This concept greatly simplifies the power, signal and thermal interfaces for the power electronics. The current sensor is used to measure and monitor the phase currents of the electric motor. To withstand the extremely harsh EMC environment in this application Sensitec developed a shielded current sensor with a specially shaped bus bar to allow easy integration within the IBB (Figure 15).

This sensor incorporates the CFS1000 sensor described above. Figure 16 shows the dynamic response of the modified MR-based current sensor, which is a factor of 4 faster than a hall-based alternative sensor.

Fig. 15: Modified MR-based current sensor for integration in inverter building block

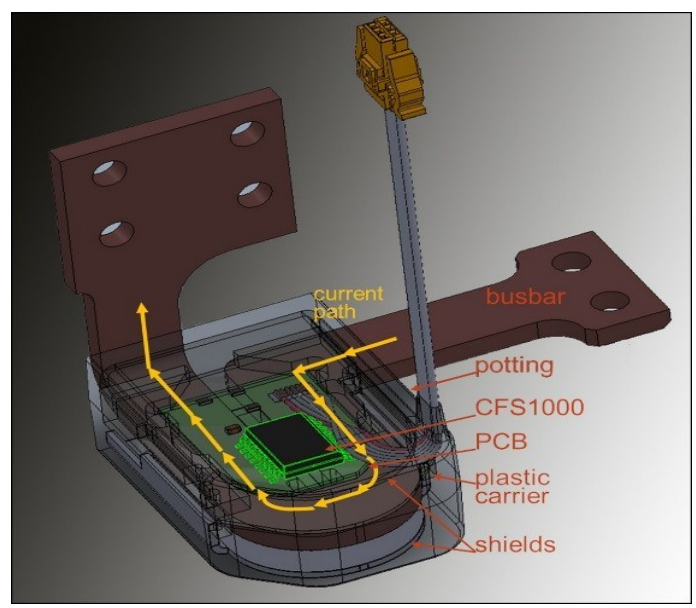

The specially modified current sensor also exhibits highly linear characteristics over the full +/- 500 A current range. Without shields the accuracy of the current sensor was negatively influenced by the extremely strong stray fields. However, the additional shielding successfully supressed these errors and provided a hysteresisfree performance which was far superior to alternative hall-based sensors (Figure 17).

Fig. 16: Dynamic response of MR- and hall-based current sensors

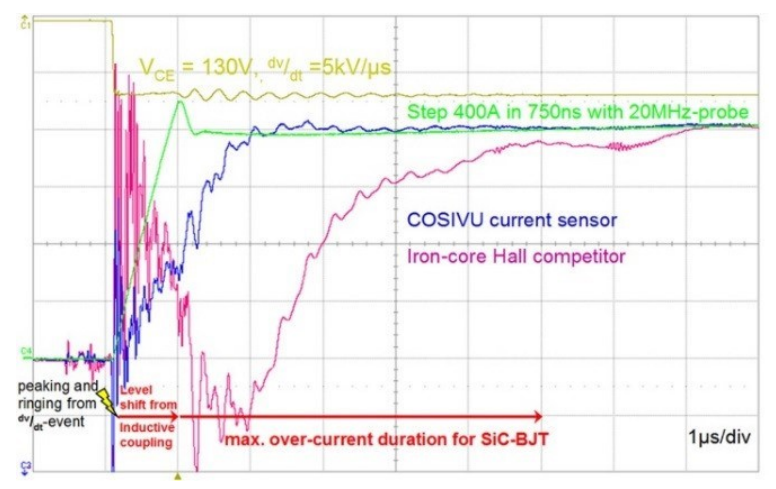

Fig. 17: Characteristic curves for MR- and hall-based current sensors

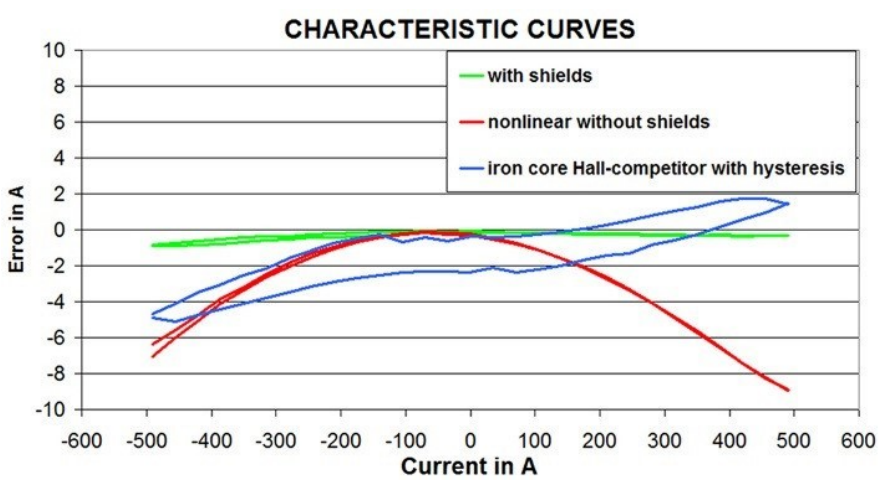

\section{Outlook}

The application area for MR-based current sensors in the field of power electronics is growing continuously. The high bandwidth allows higher switching frequencies and thereby the full exploitation of the potential of wide bandgap devices to increase the power density of drive inverters and DC/DC converters in the electrification of vehicles. MRbased sensors are not only being applied increasingly in automotive traction applications [7], but also in power electronics for aircraft [8] as well as for spacecraft [9]. In all these application areas the trend to increasing power density is accelerating. 
The high bandwidth not only enables higher power density for power electronics, but can also lead to more compact actuators as well. Fast and precise current measurement permits the use of advanced control techniques, e.g. for sensorless control of synchronous motors, which can avoid the need for a rotor position sensor on the motor. Oversampling of the motor current allows a low noise calculation of the rate of change of current $(\mathrm{di} / \mathrm{dt})$, which improves the measurement resolution and allows smooth operation down to zero speed without negative side-effects, such as acoustic noise. [10], [11].

\section{References}

1. Scherner, S. \& Slatter, R., New applications in power electronics for highly integrated high-speed magnetoresistive current sensors, Proc. of 8th International Conference on Integrated Power Electronic Systems - CIPS, Nuremberg, 2014

2. März, M., Lösungen für lokale Gleichstromnetze - $\quad$ Fraunhofer IISB zeigt Neuheiten auf der PCIM Europe 2014, Press Release, Fraunhofer IISB, May 2014

3. Eckardt, B., Effizienz an Bord - Ein Bündnis aus Forschung und Industrie entwickelt miniaturisierte Leistungselektronik für Flug- und Kraftfahrzeuge, Press Release, Fraunhofer IISB, July 2011

4. Otto, A. et al, Reliability for new SiC BJT Power Modules for Fully Electric Vehicles, 18th International Forum on Advanced Microsystems for Automotive Applications (AMAA), Berlin, 23.-14.6.2014

5. Andersson, A, COSIVU - Compact, Smart and Reliable Drive Unit for Commercial Electrical Vehicles, 18th International Forum on Advanced Microsystems for Automotive Applications (AMAA), Berlin, 23.-14.6.2014

6. Nord, S., Hilpert, F. \& Brusius, M., Integration of Magnetoresistive Current Sensors into the COSIVU Smart Drive System, Proc. of 13th MR-Symposium, Wetzlar, 2015

7. Mühlfeld, o. et al, Automotive Traction Inverter for Highest Power Density, Proc. of PCIM Europe 2015 Conference, Nuremberg, 2015

8. Kaiser, K. et al, Design of a lightweight DC/DC converter providing fault tolerance by series connection of low voltage sources, Proc. of 1st Aerospace Sensors Conference, Frankfurt / Main, 2012

9. Goffin, B., Hermetic packaging of an AMR current sensor for space applications, Proc. of 13th Symposium for Magnetoresistive Sensors and Magnetic Microsystems, Wetzlar, 2015

10. Slatter, R., High bandwidth current sensors as an enabler for advanced control techniques, Proc. of 4th International Conference on Electric Drives Production - EDPC, Nuremberg, 2014

11. Landsmann, P. \& Kennel, R., Rotor Position Estimation based on Current Oversampling, Proc. of 13th MRSymposium, Wetzlar, 2015 